Research Article

\title{
Microbiology and antibiotic sensitivity of odontogenic space infection
} Santosh $A N^{1}$, Viresh $A N^{2}$, Sharmada $B K^{3}$

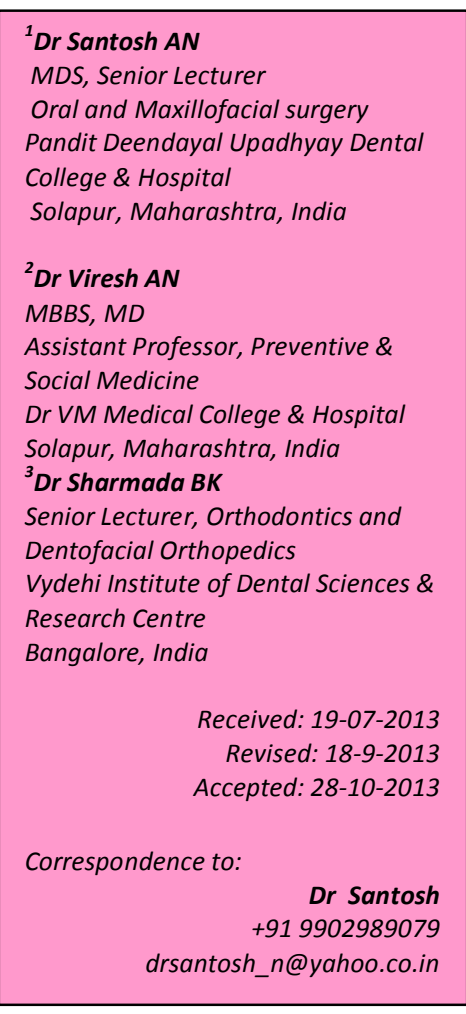

\section{Introduction}

There have been recent dramatic improvements in the morbidity and mortality associated with infectious diseases, microorganisms have proved to be quite adaptable, displaying an unsettling ability to re-emerge in continuing cycles of disease and have adapted to every environmental niche on the planet and are now adjusting to a world laced with antibiotics. ${ }^{[1]}$ Dental infection has plagued humankind for as long as our civilization exists. Hardly any imagination is required to picture a primitive man suffering pain and swelling of the face due to an abscess tooth, the cause of which could be due to periodontal trauma or the treatment. the anaerobes.
ABSTRACT
Background: Dental infections, including gingivitis, odontogenic infections periodontitis, dental caries and, result in frequent dental visits. Infection can be mild buccal space infection or severe life threatening multi space infection.

jectives: To appraise causative microorganisms responsible for odontogenic

Materials and Methods: 90 patients with orofacial space infections were in the department of microbiology for culture and antibiotic sensitivity.

Results: Aerobic organisms were highly sensitive to Ceftriaxone 95.2\%, Levofloxacin $90.5 \%$ and Amoxicillin and Clavulanic acid for $81 \%$ and were resistance to Ampicillin and Cefaclor 47.6\%. Anaerobic organisms were $100 \%$ resistance to Ampicillin and were $100 \%$ sensitive to Cephalothin, Cephalexin, Clavulanic acid. Ampicillin resistance was seen in $47.6 \%$ of aerobes and $100 \%$ of

Conclusion: This study confirms that the microbiological flora of odontogenic infections consists of complex mixture of aerobic and anaerobic bacteria. The microorganisms isolated were Streptococcus viridians, Klebsiella, Pseudomonas Aeruginosa, and Coagulase negative Staphylococci. Most common anaerobic organisms were Peptococci and Peptostreptococci. Amoxicillin/clavulunic acid and Cefotaxime were most effective antibiotics.

Keywords: Odontogenic infections, streptococcus viridians, amoxicillin, clavulunic acid, ceftriaxone

dental caries. Indeed, infection due to dental origin is one of the most common diseases of human beings and in underdeveloped countries this could be a frequent cause of death. The remains of Pre Columbian Indians, unearthed in the Midwest, and the remains of people who lived in early Egypt have revealed the bony crypts due to dental abscesses, sinus tracts, and the ravages of osteomyelitis of the jaws. ${ }^{[2]}$

Infections in the orofacial region are known to be commonly of dental origin. Odontogenic infections are one of the most frequently occurring infectious processes known to both antiquity and present day health practice. Most odontogenic 
infections arise as a sequel to pulpal necrosis caused by caries, trauma, periodontitis etc. Odontogenic infections range from periapical abscesses to superficial and deep infections in the neck. ${ }^{[1]}$

Truly we live in the "antibiotic era." Beginning with early work of Sir Alexander Fleming in 1929, when penicillin become the first "miracle drug," innumerable lives have been saved from such scourges as pnenumonia, wound sepsis, and bacteria. ${ }^{8}$ The availability of the "Wonder drug" penicillin in the year immediately after World War II ushered in a new era of complacency in infectious disease management. Many traditional management techniques such as isolation, quarantine and scrupulous application of aseptic methods in the office and operatory were prescribed when symptoms initially appeared, without determining either the cause of the disease or antibiotic susceptibility of the microbe. [2] The microbial of orofacial odontogenic infections has been researched many times and reported frequently in literature. On reviewing the available literature it has been observed that the nature of infections generally is found to be polymicrobial. Various bacteriological studies show variation in their conclusions. ${ }^{[2]}$

Anaerobic bacteria are prevalent in orofacial, head and neck infections, although it has been difficult to investigate the in vivo pathogenic potential of individual species because they are also part of the normal oralflora. Furthermore, as commensal organisms anaerobes are subjected throughout life to a continuous challenge by antimicrobial agents used in clinical therapy, resulting in selection of resistant strains. Because the emergence of clinically significant antimicrobial resistance may complicate the outcome of head and neck infections, the susceptibility patterns of anaerobes have profoundly influenced therapeutic decisions in this context in recent years, with a major impact on the antimicrobial therapy of orofacial odontogenic infections. ${ }^{[2]}$

As, commensal organisms are subjected, throughout life to a continuous challenge by antimicrobial agents used in clinical therapy, resulting in resistant strains. The emergence of clinically significant antimicrobial resistance may complicate the outcome of head and neck infections. The study of microbiologic flora and antimicrobial susceptibility will help clinician to establish the efficacy of any particular antibiotic to combat orofacial infections of odontogenic origin. The knowledge of common pathogens and their resistance status guide the Clinician towards appropriate selection of empirical antibiotics. ${ }^{[2]}$

This study has been designed to obtain valuable information of the laboratory data regarding the microbiology and antimicrobial susceptibility of microorganisms causing odontogenic infections which will help to formulate proper treatment plan for the benefit of the patient.

The objectives of the study were to identify the specific microorganisms causing the infection, to identify the resistant microorganisms causing the infection, also to quantify the role of aerobic and anaerobic organisms in causing orofacial infections, and to provide a better perspective in management of odontogenic infection. 


\section{Material and Methods}

The Study was conducted

in our SMILES...S For Life, Multispecialty Dental Health Care Center and other Dental Health Care Centers across Bengulru over the period of two years. 90 adult patients with orofacial space infections were recruited in the study after satisfying inclusion and exclusion criteria.

Inclusion Criteria: All diagnosed cases (moderate to severe infections in the form of abscess in the oro-facial region) between 18 to 60 years of age (both genders) who are willing to participate in the present study.

Exclusion Criteria: Cases below 18 yrs and above 60 yrs of age (both genders), those are not willing to participate in the study and have not given written consent, patients with known hypersensitivity to antibiotics and or with prior antibiotic administration, diabetic patient's and pregnant females.

Method of specimen collection: Pus was collected by asprirating abscess using sterile 18 gauze disposable needles with $5 \mathrm{ml}$ disposable syringes introaorally or extrorally maintaining asepsis. Also sample was collected on Swab Sticks, following all the aseptic precautions including irrigation with $0.2 \%$ chlorehixidne for intraoral sites and the skin cleaned with Povidone iodine $5 \%$ solution and alcohol for extra oral sites.

The aspirated content was immediately empted into Robertson's Cooked Meat Media and then the transport media \& swab stick was transported to the Department of Microbiology \& Histopathology for culture and antibiotic susceptibility testing. Pus samples were processed and smear studies of gram staining was made and reported.

For aerobic culture the samples were inoculated on blood agar, Mac-Conveys agar, and Nutrient Broth. Incubation was done aerobically at $37^{\circ} \mathrm{C}$ for 18 to 24 hours. The colonies were identified by grams staining and biochemical tests. For gram positive coccid Catalase Backtracking sensitivity, Opt chin sensitivity, Coagulase test and growth in $6.5 \%$ sodium chloride were used. For gram negative bacilli Oxidase test, Catalase test, Insole test, Unease test, Citrate test and Triple sugar iron were used. If no growth was observed after the first culture, subcultures from nutrient broth was made on Mac -Coney's agar, Blood agar and looked for growth after overnight incubation. Growth was identified in UV protected Laminar Floor (Fig.1) using appropriate biochemical test.

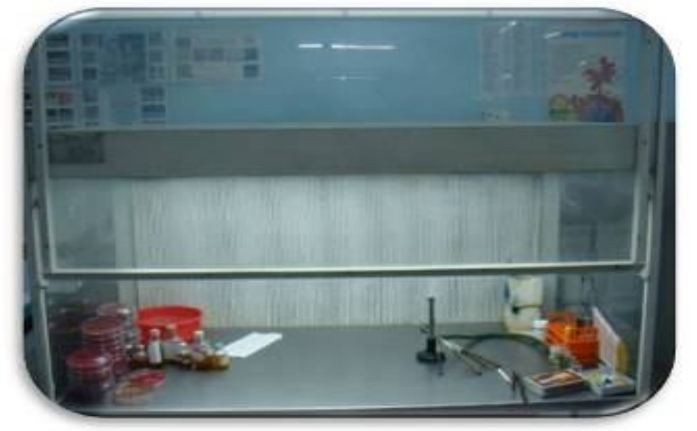

Fig. 1 Laminar Floor UV protected

For anaerobic culture, sample was inoculated into plain blood agar and streaked. A filter paper disk containing Amoxicillin and Clavulanic acid was incorporated on the blood agar after streaking for anaerobic culture. These plates were kept inside $\mathrm{Hi}$ an aero gas pack jar (Fig.2) $\mathrm{Hi}$ - media with gas pack and incubated at $37^{\circ} \mathrm{c}$ for 48 hours to 72 hours. 


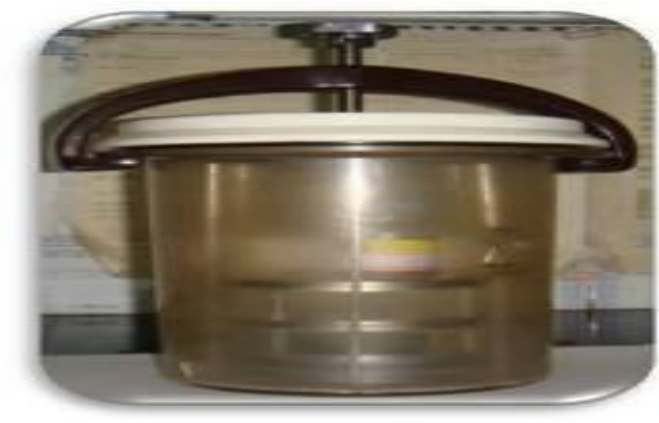

Fig. 2 Anaerobic gas jar

The plates were observed for colony formation. The colonies were identified by gram's stain morphology, haemolysis, and sensitivity to antibiotics like Amoxicillin and Clavulanic Acid (Augmentin), Vancomycin, Colistin, Growth in bile, Indole test, Pigmentation, Lipase, Catalase and Sodium Polyethanol Sulphonate, If no growth was observed after first culture, subculture was done from Roberston cooked meat broth on plain blood agar and identified as mentioned above.

Antibiotic sensitivity test for the isolates were performed in Muller Hinton agar by disc diffusion method of Kirby Bauer. The zone of inhibition were measured and recorded as sensitive, intermediate sensitive and resistant as indicated in the Kirby - Bauer method. The antibiotic discs were obtained from $\mathrm{Hi}$ Media. (Fig 3, 4)

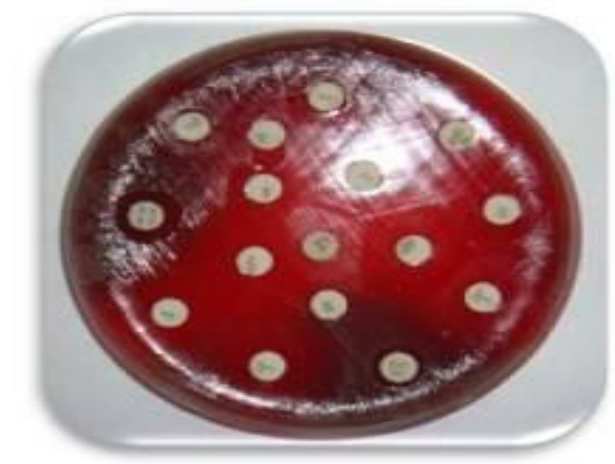

Fig. 3 Antibiotic sensitivity of Aerobic bacteria

IJMDS • www.ijmds.org • January 2014; 3(1)

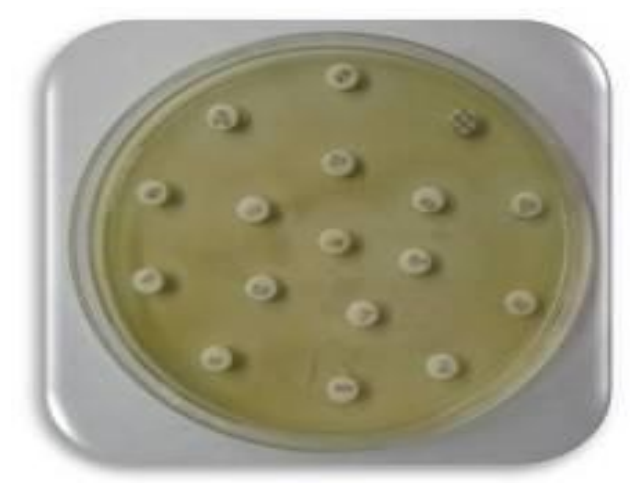

Fig. 4 Antibiotic sensitivity of anaerobic bacteria

Causative teeth were identified by clinical \& radiological examination and were immediately extracted or were sent for endodontic treatment. Incision and drainage by Hilton's method was carried out under local anesthesia with proper aseptic precautions on all the patients. The corrugated rubber drain was placed if required and stabilized with sutures. Drain was kept in place for less than 48 hours so as to prevent accumulation of fluid ( blood, pus and infected fluid and also to prevent accumulation of air (dead space) All patients were started with empirical antibiotics in the form of Amoxicillin / Clavulanic acid $625 \mathrm{mg} \mathrm{PO} Q 8 \mathrm{H}$ and Metronidazole PO 400mg TID Q8H. For severe ill patients like airway compromise, aspiration risk, cutaneous fistulas and necrotizing fasciitis patients were started with Inj. Cefotaxime IV $1 \mathrm{~g}$ Q $12 \mathrm{H}$ and Inj. Metronidazole IV 500mg Q8H.After cultures and sensitivity, depending on the clinical course of the disease appropriate antibiotics were given for 7 to 10 days.

\section{Results}

In the present study 90 patients with orofacial odontogenic space infections were considered. Most commonly involved age group was between 29 to 39 years, 57 
$(63.33 \%)$ cases were males while 33 (36.67\%) cases were females; showing males were more prone to oro-facial odontogenic space infections as compared to females. The bacteria were found to be

Table 1: Types of bacteria in grams stain smear study
33(31.4\%) gram positive aerobic, there were $39(37.1 \%)$ gram positive anaerobic and 33 (31.4\%) gram negative aerobic as shown. (Table 1)

\begin{tabular}{|c|c|c|}
\hline Bacteria & Frequency & $\%$ \\
\hline Gram positive aerobic & 33 & 31.4 \\
\hline Gram negative aerobic & 33 & 31.4 \\
\hline Gram positive anaerobic & 39 & 37.1 \\
\hline Gram negative anaerobic & 00 & 0.0 \\
\hline Total & 105 & 100 \\
\hline
\end{tabular}

In aerobic organisms Streptococcus Viridans were seen in 24 (36.4\%), Coagulase negative Staphylococci were seen in 06 (9.1\%), Klebsiella in 18 (27.3\%), Pseudomonas Aeruginosa in 12 (18.2\%), Enterobacter Spp in 03 (4.54\%), Neisseria 03 (4.54\%). (Fig. 5)

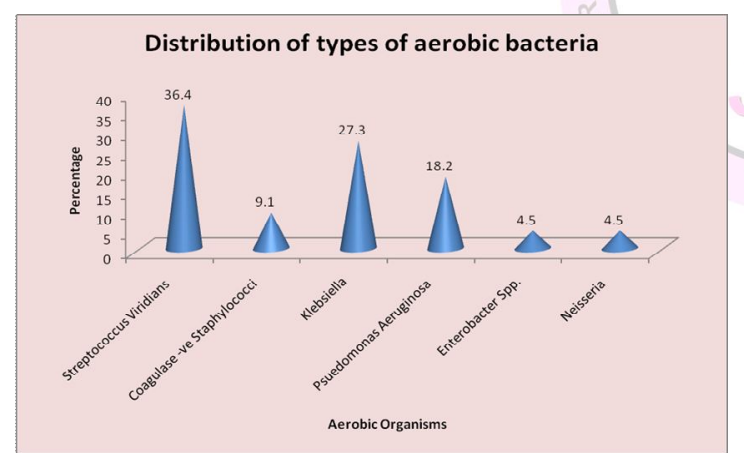

Fig.5 Distribution of types of aerobic bacteria $(n=66)$

In anaerobic organisms Peptococci were seen in 23 (58.9\%) and Peptostreptococcus in 16 (41.1\%). (Fig. 6) In the site distribution of orofacial odontogenic infections, the most common site was Buccal Space, followed by Canine space. Right side buccal space was more common than left side. (Fig.7)

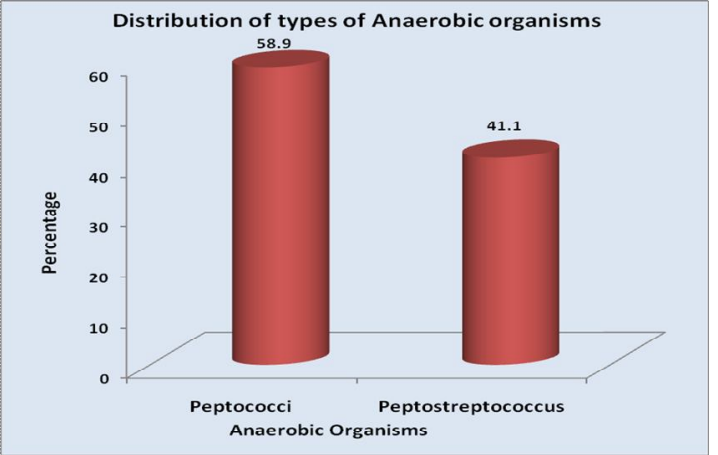

Fig. 6 Distribution of types of anaerobic bacteria $(n=39)$

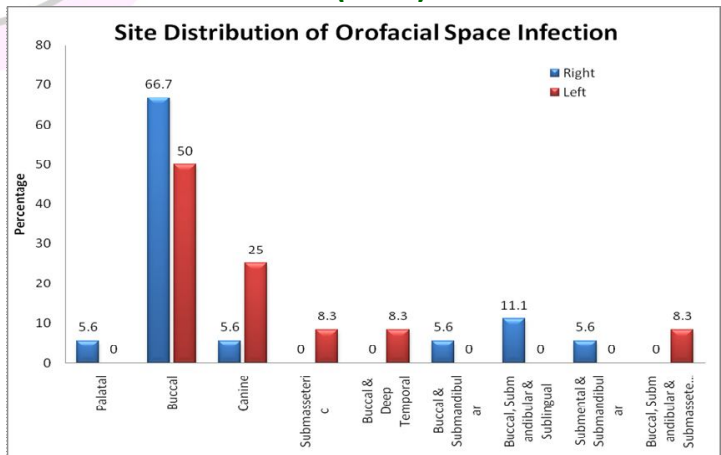

Fig. 7 Site Distribution of Orofacial Space infections

Aerobic bacteria were sensitive to Ceftriaxone, followed by Levofloxacin, Amoxicillin and clavulanic acid. Antibiotic Resistance seen in Aerobic bacteria was for Ampicillin and Cefaclor, followed by Cefazolin. (Table 2) 
Santosh et al: Odontogenic space infection

Table 2: Antibiotic sensitivity pattern of Aerobic strains ( $N=66$ )

\begin{tabular}{|c|c|c|c|c|}
\hline Antibiotics & Sensitivity & $\%$ & Resistance & $\%$ \\
\hline Amoxicillin / Clavulanic acid (Augmentin) & 53 & 80.3 & & \\
\hline Azetreonam & 03 & 4.5 & & \\
\hline Ampicillin & 09 & 13.6 & 10 & 47.6 \\
\hline Amikacin & 22 & 33.3 & & \\
\hline Cephalothin & 35 & 53.0 & & \\
\hline Cephalexin & 35 & 53.0 & & \\
\hline Cotrimoxazole & & 0.0 & 03 & 4.5 \\
\hline Cefemine & 03 & 4.5 & & \\
\hline Cefazolin & & 0.0 & 28 & 42.4 \\
\hline Cefaclor & 03 & 4.5 & 10 & 47.6 \\
\hline Cefuroxime & 22 & 33.3 & 06 & 9.1 \\
\hline Cefaperazone / Sulbactam (Magnex) & 35 & 53.0 & & \\
\hline Cefaperazone & 09 & 13.6 & & \\
\hline Ceftazidime & 38 & 57.6 & & \\
\hline Cefotaxime & 10 & 47.6 & 02 & 9.5 \\
\hline Ceftriaxone & 63 & 95.4 & & \\
\hline Imipenem / Cilastatin (Cilanem) & 03 & 4.5 & & \\
\hline Meropenem & 03 & 4.5 & & \\
\hline Clindamycin & 25 & 37.8 & & \\
\hline Erythromycin & 35 & 53 & & \\
\hline Tobramycin & 13 & 19.7 & & \\
\hline Gentamicin & 13 & 19.7 & & \\
\hline Ciprofloxacin & & 0.0 & 03 & 4.5 \\
\hline Gatifloxacin (Gatiquin) & 50 & 75.7 & & \\
\hline Linezolid & 35 & 53 & & \\
\hline Levofloxacin & 60 & 90.9 & & \\
\hline Methicillin & 28 & 42.4 & & \\
\hline Ticarcillin / Clavulanic acid (Timentin) & 38 & 57.5 & & \\
\hline $\begin{array}{c}\text { Piperacillin/Tazobactam } \\
\text { (Tazact) }\end{array}$ & 35 & 53 & & \\
\hline Teicoplanin (Targocid) & 31 & 47.1 & 03 & 4.5 \\
\hline Vancomycin & 31 & 47.1 & 03 & 4.5 \\
\hline
\end{tabular}


Among the Anaerobic bacteria it was found that most of the organisms were sensitive for Clindamycin, Erythromycin, Gatifloxacin, Cephalothin, Cephalexin followed by
Amoxicillin and Clavulanic acid. Antibiotic Resistance seen in anaerobic bacteria was for Ampicillin and Azetreonam. (Table 3)

Table 3: Antibiotic sensitivity pattern of anaerobic strains $(n=39)$

\begin{tabular}{|c|c|c|c|c|}
\hline Antibiotics & Sensitivity & $\%$ & Resistance & $\%$ \\
\hline Amoxicillin / Clavulanic acid (Augmentin) & 36 & 92.3 & & \\
\hline Azetreonam & & & 03 & 7.7 \\
\hline Ampicillin & & & 39 & 100.0 \\
\hline Cephalothin & 39 & 100.0 & & \\
\hline Cephalexin & 39 & 100.0 & & \\
\hline Cefixime & 06 & 15.4 & \\
\hline Ceftriaxone & 36 & 92.3 & \\
\hline Ciprofloxacin & 03 & 7.7 & \\
\hline Gentamycin & 06 & 15.4 & & \\
\hline Clindamycin & 39 & 100.0 & & \\
\hline Erythromycin & 39 & 100.0 & & \\
\hline Gatifloxacin (Gatiquin) & 39 & 100.0 & & \\
\hline Linezolid & 39 & 100.0 & & \\
\hline Levofloxacin & 39 & 100.0 & & \\
\hline Methicillin & 06 & 15.4 & & \\
\hline Clavulanic acid (Timentin) & 10 & 25.6 & & \\
\hline Piparcillin / & 39 & 100.0 & & \\
\hline Teicoplanin (Targocid) & 03 & 7.7 & \\
\hline Vancomycin & 39 & 100.0 & & \\
\hline
\end{tabular}

\section{Discussion}

Odontogenic infections are typically polymicrobial. The pathogenesis of odontogenic infections is dependent on a synergistic relationship between aerobic and anaerobic bacteria. The last decade has seen a notable change in the behavior of odontogenic infections. The severity of these infections is far greater than in the past, demanding swift recognition of the disease followed by prompt, and more aggressive treatment. Failing to identify and treat these infections promptly may result in disastrous outcomes, as occurred in a recently sensationalized case entitled "Death of a Toothache Victim," which was reported in March 1998 in the Toronto Star. [4]

In our study thirty patients with orofacial odontogenic space infections were considered. The age group most commonly involved was between 29 to 39 years. This finding is comparable to the age distribution reported by Loureriro FR. [4] Male patients 57(63.33\%) were more commonly than female patients 33(36.66\%). This finding can be compared to the sex distribution given by Rega AJ [8] where male patients were $54 \%$ and female patients were $46 \%$.

Most of patients were from low socioeconomic class $46.7 \%$, because these 
patients won't report to us at initial stage of caries or periapical pathology and also because of poor oral hygiene. These findings were compared with Tozoglu $\mathrm{S}^{(5)}$ in which most of the cases were from low socioeconomic class $86.8 \%$ where author has used Kuppuswamy's classification for socioeconomic class.

The most commonly involved space was Buccal space 18(60\%), followed by canine space $4(13.3 \%)$ in single space infections cases and among multiple space infection Submandibular space, Buccal space. These finding were compared with the findings of Rega $\mathrm{AJ}^{[8]}$ where they found Submandibular space (30\%), more common followed by Buccal space (27.5\%) and among multiple space Submandibular space was most common followed by Submental space. These findings were similar to the study done by Loureriro FR ${ }^{[4]}$ where Buccal mandibular space infection was most common (50\%) followed by Submandibular (31.9\%).

Mandibular first molar (31.3\%) was the most common causative tooth followed by madibular second molar, maxillary first molar and madibular third molar, probably as $1^{\text {st }}$ molars are first to erupt in the oral cavity and are more prone for decay. This study is compared with Indresano AT ${ }^{[15]}$ where third molar is most commonly involved tooth for odontogenic infections with the rate of 15:1.

In the present study most of the patients suffered from mild trismus $19(63.3 \%)$, followed by moderate $7(23.3 \%)$ and severe $4(13.3 \%)$ trismus. This study is compared with Loureriro FR ${ }^{[4]}$ where trismus was seen in $43.3 \%$ cases.

Many investigations have demonstrated that Streptococcus viridians,
Peptostrptococcus, Prevotella, Porphyromonas, and Fusobacterium are frequently isolated from odontogenic infections. $[3,11,14,16,17,18,19]$ The most common organisms isolated from aerobic bacteria were Streptococcus viridians 24 (36.4\%), followed by Klebsiella 18 (27.3\%), Pseudomonas Aeruginosa 12 (18.2\%), Coagulase negative Staphylococci 6 (9.1\%), Nisseria 3 (4.54\%) and Enterobacter Spp $3(4.54 \%)$. The most common organisms isolated from anaerobic bacteria were Peptococci $23 \quad$ (58.9\%) and Peptostreptococci 16 (41.1\%). This study was compared with Rega AJ ${ }^{[8]}$ in which Viridians Streptococci were the predominant species, followed by Provetella, Staphylococci and Peptostreptococcus. A high rate of Staphylococci (8.9\%) was cultured from the total isolates, which may be due to contaminant of cultures from the skin or an actual finding. The prevalence of bacterial species varies, with multiple studies demonstrating Streptococci Viridans their predominant species and other studies that show predominance of Gram-negative rods (Bacteroides/Provetella).The prevalence of bacterial species varies, with multiple studies demonstrating Streptococcus viridians as their predominant species $[10,23,24]$ and other studies that show predominance of gram negative rods (Bacteroides/Prevotella). [12, 16, 22] Many studies have demonstrated that Prevotella, Porphyromonas and Fusobacterium are the predominant bacteria among anaerobic gram negative rods isolated from orofacial odontogenic infections. ${ }^{[11,16,19]}$

In the present study all the aerobic bacteria showed $3(14.3 \%)$ sensitive to Ampicillin and $10(47.6 \%)$ resistance to the 
same. Most of the organisms were sensitive to Ceftriaxone 20(95.2\%), Levofloxacin $19(90.5 \%)$ and $17(81 \%)$ sensitive for Amoxicillin and Clavulanic acid. Aerobic bacteria were resistance to Cefazolin 9(42.9\%) and 10(47.6\%) resistance to Cefaclor. Amoxicillin / Clavulanic acid (Augmentin) were $100 \%$ effective against Peptostreptococcus and Staphylococcus aureus. So when staphylococcus aureus infection is suspected these antibiotics may be preferred as stated by Flynn TR ${ }^{[6]}$ which has been in this study also. The high incidence of Staphylococcus aureus and pseudomonas Aeruginosa in this study may be a contamination of skin / oral micro-flora as stated by Sims. But such contamination during specimen collection is unlikely, because proper antimicrobial skin preparations were used before aspirating with disposable syringe. This shows that Amoxicillin and Clavulanic acid is still effective against aerobic bacteria which was used an empirical antibiotic in this study. This study was compared with Kuriyama T ${ }^{[6]}$ and Flynn TR. ${ }^{[7]}$

In the present study all anaerobic bacteria were resistant to Ampicillin 12(100\%). They were sensitive to Macrolide group of antibiotics like Erythromycin 12(100\%), Clindamycin $12(100 \%)$, Vancomycin $12(100 \%)$ but they were less sensitive to Methicillin 2(16.7\%), Teicoplanin 1(8.3\%). They were also sensitive to Cephalothin and Cephalexin 12(100\%), Ceftriaxone 11(91.7\%). This study was compared with Kuriyama ${ }^{[6]}$ and Flynn TR. ${ }^{[7]}$

In this study Metronidazole sensitivity was not done due to lack of Metronidazole discs in the Hospital laboratory. But Metronidazole is only effective against obligate anaerobes, because its molecule must enter the bacterial cell before it is reduced to form the active antibacterial agent and this reduction takes place effectively only under anaerobic conditions Stefaanopoulos PK. ${ }^{[9]}$

The microbiologic flora of orofacial abscess as shown in this study is polymicrobial. Microorganisms isolated were most susceptible to antimicrobial agents like Amoxicillin / Clavulunic acid, Ceftriaxone, Levofloxacillin. Majority of the anaerobes were susceptible to Levofloxacin, Gatifloxacin, Erythromycin and Ceftriaxone. Many micro-organisms isolated in this study showed increased resistance to Ampicillin.

According to literature, metronidazole is found to have high sensitivity for mixed microflora of orofacial space infection; this drug was not included in this study due to laboratory shortcomings.

Most of the organisms isolated were Aerobic with $50 \%$ growth, mixed organisms were $41.6 \%$ and pure Anaerobes were $8.3 \%$. This confirms the fact that odontogenic orofacial infections are polymicrobial. One of the canine space and buccal space infection showed purely the growth of anaerobic bacteria. In our study aerobic organisms were highly sensitive to Ceftriaxone $95.2 \%$, Levofloxacin $90.5 \%$ and Amoxicillin and Clavulanic acid for $81 \%$. They were resistance to Ampicillin and Cefaclor $47.6 \%$. In this study anaerobic organisms were $100 \%$ resistance to Ampicillin. Anaerobic organisms were $100 \%$ sensitive to Cephalothin, Cephalexin, Gatifloxacin, Linezolid and Tazact. 91.7\% were sensitive to Amoxicillin and Clavulanic acid. Metronidazole was not included in this study due to laboratory technical problems. 


\section{References}

1. Store W, Haug RH, Lillich TT. The Changing Face of Odontogenic Infections. J Oral MaxillofacSurg 2001;59:739-748.

2. Topazian, Goldberg, Hupp. Oral and Maxillofacial Infections. $4^{\text {th }}$ Edition. Philadelphia: WB Saunders company; 2002.

3. Sandor GKB, Low DE, Judd PL, Davidson RJ. Antimicrobial Treatment Options the Management of Odontogenic Infections. CDA-ADC Journal 2009;1-9.

4. Loureriro Sato FR, Casseb Hajala FA, Vasconcelos Freire Filho FW, Fernandes Moreira RW, Marico de Moraes. Eight Year Retrospective Study of Odontogenic Origin Infections in a Postgraduation Program on Oral and Maxillofacial Surgery. J Oral and MaxillofacSurg 2009;67:10921097.

5. Tozoglu S, Buyukkurt MC, Uslu H. Role of Socioeconomic Factors in Maxillofacial Abscess of Odontogenic Origin. Ataturk Univ DişHek Fak Derg, Cilt:19, Sayı: 1, Yıl: 2009, Sayfa: 26-30.

6. Kuriyama T, Karasawa T, Nakagawa K, Nakamura S, Yamamoto E. Antimicrobial susceptibility of major pathogens of orofacialodontogenic infections to 11 betalactam antibiotics. Oral Microbiollmmunol. Oct 2002;17(5):285-9.

7. Flynn TR, Shanti MR, Levi HM, Adamo AK, Kraut AR, Trieger N. Severe Odontogenic Infections, Part 1: Prospective Report. J Oral MaxillofacSurg 2006;64:1093-1103.

8. Rega AJ, Aziz SR, Ziccardi VB.Microbiology and Antibiotic Sensitivites Of Head and Neck Space Infections of Odontogenic Origin. J Oral and MaxillofacSurg 2006;64:1377-1380.

9. Stefaanopoulos PK, Kolokotronis AE. The Clinical Significance of anaerobic bacteria in acute orofacialodontogenic infections. Oral

IJMDS • www.ijmds.org • January 2014; 3(1)
Surg Oral med oral pathol Oral RadiolEndod 2004;98:398-408.

10. Storoe W, Haug RH, Lillich TT. The changing face of odontogenic infections. J Oral MaxillofacSurg 2001;59:739-48.

11. Kuriyama T, NakagawaK, KarasawaT, Saiki Y, Yamamoto E, Nakamura S. Past administration of Beta lactam antibiotics and increase in the emergence of beta lactamase producing bacteria in patients with orofacialodontogenic infections. Oral Surg Oral Med Oral Pathol Oral RadiolEndod 2000;89:186-92.

12. M Sakaguchi, S Sato, T Ishiyama, S Katsuno, $\mathrm{K}$ Taguchi. Characterization and management of deep neck infections. Int J Oral Maxillofac Surg 1997;26:131-134.

13. Sandor GK, Low DE, Judd PL, Davidson RJ. Antimicrobial treatment options in the management of odontogenic infections. J Can Dent Assoc 1998;64:508-14

14. Baker KA, Fotos PG. The management of odontogenic infections. A rationale for appropriate chemotheraphy. Dent Clin North Am 1994;38:689-706.

15. Indresano AT, Haug RH, Hoffman MJ. Third Molar as a cause of deep space infections. J Oral MaxillofacSurg 1992;50:30-5.

16. Brook I, Frazier EH, Gher ME. Aerobic and anaerobic microbiology of periapical abscess. Oral Microbiollmmunol 1991;6:123-5.

17. Gill Y, Scully C. The microbiology and management of acute dentoalveolar abscess. Views of British Oral \& Maxillofacial Surgeons. $\mathrm{Br} J$ Oral MaxillofacSurg 1988;26:452-7.

18. Lewis MA, MacFarlane TW, McGowan DA. Quantitative bacteriology of acute dentoalveolar abscesses. J Med Microbiol 1986;21:101-4. 
19. Heimdahl A, Nord CE. Treatment of orofacial infections of odontogenic origin. Scand J Infect Dis Suppl 1985;46:101-5.

20. Labriola JD, Mascaro J, Alpert B. The microbiologic flora of orofacial abscess. J Oral MaxillofacSurg 1983.

21. Hunt DE, Meyer RA. Continued evolution of the microbiology of oralinfections. J Am Dent Assoc 1983;107:52-4.

Cite this article as: Santosh AN, Viresh AN, Sharmada BK. Microbiology and antibiotic sensitivity of odontogenic space infection. Int J Med and Dent Sci 2014; 3(1):303-313.

Source of Support: Nil Conflict of Interest: No

22. Kannangara DW, Thadepalli British $H, M c$ Quiter JL. Bacteriology and treatment of dental infections. Oral Surg Oral Med Oral Pathol 1980;50:103-9.

23. Barlett JG, O'Keefe P. The bacteriology of Perimandibular space infections. J Oral Surg 1979;37:407-9.

24. Labriola JD, Mascaro J, Alpert B. The microbiologic flora of orofacial abscess. J Oral Maxillofac Surg 1983;41:711-4. 